\title{
WebSQL: Uma ferramenta de apoio ao processo de ensino e aprendizagem de SQL baseado na recomendação de objetos de aprendizagem e competências do aluno em SQL
}

\author{
Eduardo Paulo Marques Raiol, PPGCC - UFPA, marquesraiol@gmail.com \\ Igor da Penha Natal, PPGCC - UFPA, igorpnatal@gmail.com \\ Eloi Luiz Favero, PPGCC - UFPA, favero@ufpa.br
}

\begin{abstract}
Resumo. Este trabalho apresenta um AVA chamado WebSQL, baseado na recomendação de Objetos de Aprendizagem (OA) focado na avaliação, onde o sistema recomenda a avaliação (questões) mais adequada para cada aluno com base nas competências do aluno em relação a linguagem SQL, como o objetivo de servir de suporte metodológico ao processo de ensino $e$ aprendizagem de SQL, buscando tornar as aulas mais dinâmicas com a criação de um ambiente mais atraente e envolvente, onde o aluno possa sentirse motivado e encorajado a aprofundar-se nos conteúdos ministrados em sala de aula ou em laboratórios, através da realização de exercícios, atividades em grupo e avaliações, obtendo feedback automático das soluções propostas.
\end{abstract}

Palavras-chave: SQL, Sistema de Recomendação, Objetos de Aprendizagem, Competências, Ambiente Virtual de Aprendizagem

\section{WebSQL: A tool to support the teaching and learning process $S Q L$ based on the recommendation of learning objects and powers of the student in $S Q L$}

\begin{abstract}
This paper presents a AVA called WebSQL, based on the Learning Object Recommendation (OA) focused on evaluation, where the system recommends evaluating (issues) most appropriate for each student based on the student's skills in relation to SQL language, such as to serve as a methodological support to teaching and learning SQL, helping to make the classes more dynamic by creating a more attractive and engaging environment where the student can feel motivated and encouraged to deepen the content taught in classroom or laboratory, by performing exercises, group activities and assessments, obtaining automatic feedback of the solutions proposed.
\end{abstract}

Keywords: SQL, the Recommendation System, Learning Objects, Skills, Virtual Learning Environment

\section{Introdução}

Segundo Lazzaroto et al. (2011), a informática tem sido considerada, pela maioria dos profissionais da educação, psicologia e da ciência da computação uma grande aliada no processo de ensino e aprendizagem, porém, tais pesquisas têm demonstrado que o uso da informática na educação somente será eficaz com o desenvolvimento de ambientes 
educacionais, onde o computador seja utilizado como uma ferramenta de interação entre o aluno, o professor e o conteúdo a ser ensinado.

Assim como mostram Pereira e Rapkiewicz (2004), a grande importância de se aprender algoritmos para criar programas de computador, verifica-se também que é fundamental o aluno aprender a Linguagem de Consulta Estruturada (SQL), do inglês Structured Query Language, para recuperação de dados. Logo, acredita-se, que é fundamental proporcionar ao aluno um ambiente onde ele possa praticar listas de exercícios e realizar avaliações práticas, que segundo Prior e Lister (2004) a habilidade em programação de uma nova linguagem não pode ser adquirida sem um esforço significativo nas atividades práticas.

Neste contexto, a utilização de Ambientes Virtuais de Aprendizagem (AVA) que forneçam suporte a realização de atividades práticas, apresenta um grande potencial para o processo de ensino e aprendizagem como um todo, em especial da linguagem SQL, tornando as aulas mais dinâmicas e buscando criar um ambiente mais atraente e envolvente, onde o aluno possa sentir-se motivado e encorajado a aprofundar-se nos conteúdos ministrados em sala de aula ou em laboratórios.

Este trabalho apresenta um AVA chamado WebSQL, baseado na recomendação de Objetos de Aprendizagem (OA) focado na avaliação, onde o sistema recomenda a avaliação (questões) mais adequada para cada aluno com base nas competências do aluno em relação a linguagem SQL, para apoiar o processo de ensino e aprendizagem de SQL, possibilitando a realização de exercícios, atividades em grupo e avaliações, com feedback em tempo real das soluções propostas pelos alunos, motivando assim cada vez mais o aluno a buscar soluções ótimas para suas consultas ao Banco de Dados.

\section{Modelando o Perfil do Aluno baseado nas suas Competências em SQL}

Fleury e Fleury (2001) apresentam o conceito de competência como sendo uma característica subjacente a uma pessoa que é casualmente relacionada com desempenho superior na realização de uma tarefa ou em determinada situação. Já Perrenoud (1999), define competência como a faculdade de mobilizar um conjunto de recursos cognitivos (saberes, capacidades, informações, etc.) para solucionar com pertinência e eficácia uma série de situações. Para o contexto deste trabalho entende-se competência como a capacidade que o aluno tem em realizar consultas, complexas ou não, ao banco de dados, utilizando a linguagem SQL.

\subsection{Criação e Manutenção do Perfil do Usuário}

Um perfil ou modelo de usuário consiste, principalmente, de conhecimento sobre as preferências individuais que determinam o comportamento do usuário. Entende-se por preferências todas aquelas informações que são diretamente necessárias para a adaptação do comportamento do sistema aos interesses do usuário (Kobsa, 2001 citado por Barth, 2010).

O perfil do usuário é adquirido e atualizado, no WebSQL, de duas formas, respectivamente; explícita, através da aplicação de questionário, com utilização do algoritmo de aprendizado de máquina KNN para classificação inicial do aluno e implícita, onde o sistema monitora as interações do usuário com o ambiente, para então atualizar seu perfil. 
Na explícita, o aluno ao acessar o WebSQL pela primeira vez, é direcionado para um formulário de cadastro de competências em SQL, contendo perguntas acerca de grupos de conhecimentos, que serão necessárias para o sistema classificar o aluno em determinado nível de conhecimento e gerar seu perfil inicial, conforme figura 01.

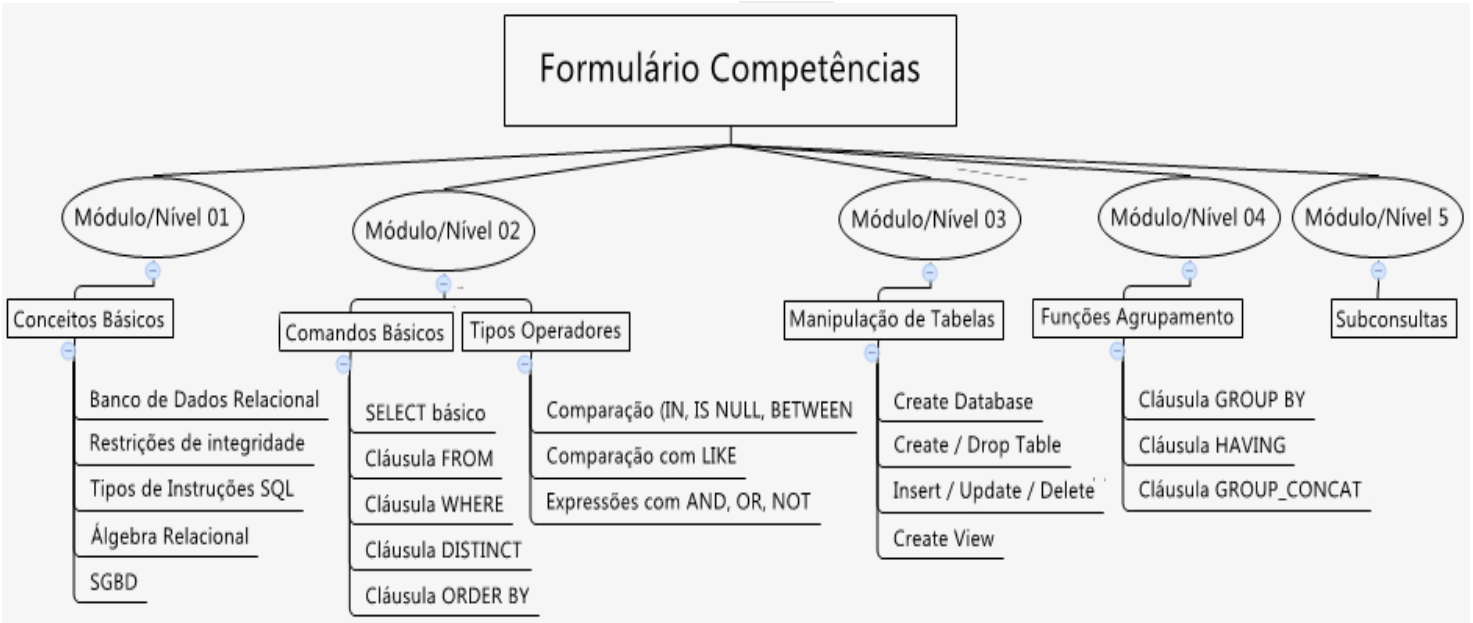

Figura 01: Grupo de questões para o cadastro de competências

Na figura 02, podemos visualizar o questionário sendo aplicado pelo sistema para captação das informações que servirão para criação do perfil inicial.

\begin{tabular}{|c|c|c|}
\hline Websil & $\begin{array}{l}\text { Universidade Federal do Pará } \\
\text { Programa de Pós Graduaçăo em Ciência da Computação } \\
\text { WebSql - Ferramenta de Apoio ao Ensino / Aprendizagem em SQL }\end{array}$ & Jedow \\
\hline \multicolumn{2}{|l|}{ त̂̀ Menu do ANDRÉ MIGUEL PENA BAHIA } & Sair do WEBSQL \\
\hline \multicolumn{3}{|l|}{ CADASTRO DE COMPETÊNCIAS EM SQL } \\
\hline \multirow{6}{*}{\multicolumn{3}{|c|}{$\begin{array}{l}\text { CRIENTAÇÖES } \\
\text { CARO ALUNO, O objetivo desse formulário é classificá-lo em determinado nível de competência } \\
\text { (conhecimento) em SQL. Preste atenção aos itens questionados e responda apenas "SIM", se possui } \\
\text { conhecimento ou "NÃO", se não possui conhecimento acerca de cada item que está sendo perguntado, } \\
\text { conforme o grupo de domínio. }\end{array}$}} \\
\hline & & \\
\hline & & \\
\hline & & \\
\hline & & \\
\hline & & \\
\hline \multicolumn{2}{|l|}{ Q.01 - CONCEITOS BÁSICOS } & 相 \\
\hline \multicolumn{2}{|l|}{ Q.02 - COMANDOS BÁSICOS EM SQL } & 面 \\
\hline \multicolumn{2}{|l|}{ Q.03 - TIPOS DE OPERADORES } & 目 \\
\hline \multicolumn{2}{|l|}{ Q.04 - MANIPULAÇÄO DE TABELAS } & 目 \\
\hline \multicolumn{2}{|l|}{ Q.05 - FUNÇÖES DE AGRUPAMENTO } & 相 \\
\hline GRAVAR VOLTAR & & \\
\hline
\end{tabular}

Figura 02: Questionário para o cadastro de competências no WebSQL 
Após o cadastro de competências do aluno, o sistema, com base nas informações cadastradas, classifica o aluno em determinado módulo/nível de conhecimento em SQL, conforme a árvore de classificação da figura 03.

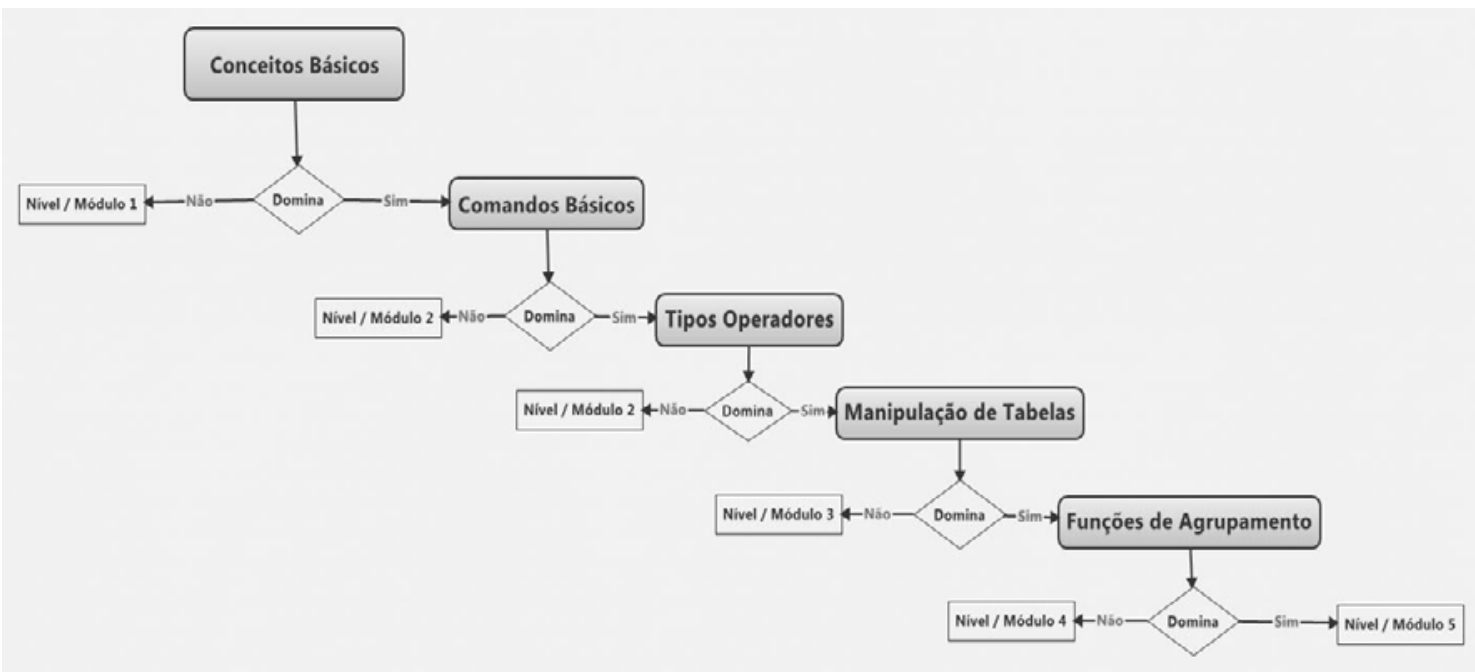

Figura 03: Árvore de classificação do aluno por nível de competência

Para ser feita a classificação dos usuários de forma automática pelo sistema, foi utilizado o algoritmo de aprendizagem de máquina KNN, onde busca por similaridades em atributos para identificar novos integrantes do grupo. A base de treino utilizada contém 117 instâncias e 21 atributos que correspondem as 20 questões do formulário e 1 para a classe (níveis/módulos). Essa base foi feita de acordo com interação junto ao especialista para a classificação em cada nível. O primeiro teste para validar o modelo foi utilizando o cross-validation com valor 10, onde foi possível obter pouco mais de 92\% de acerto do classificador, conforme mostra a diagonal principal da figura 04 .

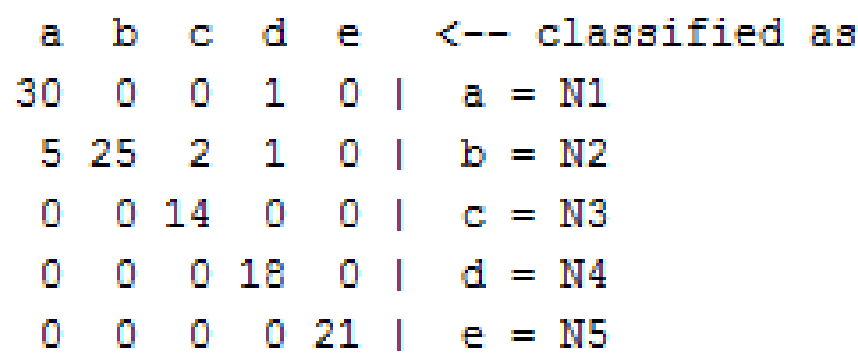

\section{Figura 04: Resultado do classificador utilizando Cross-Validation na base de treino}

Foram coletadas respostas de 10 usuários do questionário de classificação. Primeiro foi verificado manualmente em que nível cada usuário se encaixaria, e depois foi validado através do modelo apresentado anteriormente, onde obteve-se $90 \%$ de acerto, mais uma vez comprovando a eficiência da base de treino. O que é importante observar, através da figura 05, é que o único erro do classificador foi a classificação do usuário em um nível acima, ou seja, mesmo errando ele conseguiu colocar próximo ao resultado esperado. 


$\begin{array}{lllllll}\mathrm{a} & \mathrm{b} & \mathrm{c} & \mathrm{d} & \mathrm{e} & <-- \text { classified as } \\ 3 & 1 & 0 & 0 & 0 & \mathrm{a} & \mathrm{a}=\mathrm{N} 1 \\ 0 & 2 & 0 & 0 & 0 & \mathrm{|} & \mathrm{b}=\mathrm{N} 2 \\ 0 & 0 & 0 & 0 & 0 & \mathrm{|} & \mathrm{c}=\mathrm{N} 3 \\ 0 & 0 & 0 & 4 & 0 & \mathrm{I} & \mathrm{d}=\mathrm{N} 4 \\ 0 & 0 & 0 & 0 & 0 & \mathrm{a} & \mathrm{e}=\mathrm{N} 5\end{array}$

Figura 05: Resultado do classificador utilizando a base de teste

Uma vez gerado o perfil inicial do aluno, a manutenção desse perfil se dará de forma implícita (automática), através do monitoramento das atividades do aluno no WebSQL. Serão somadas todas as pontuações obtidas pelo aluno em todas as atividades e avaliações, do nível atual do aluno, realizadas no sistema, bem como as pontuações das atividades e avaliações propostas, se a soma das pontuações do aluno for maior ou igual a $70 \%$ do total dos pontos das atividades e avaliações, o sistema atualiza seu perfil, classificando o aluno no nível/módulo exatamente superior ao que encontra-se no momento da avaliação, ver figura 06 .

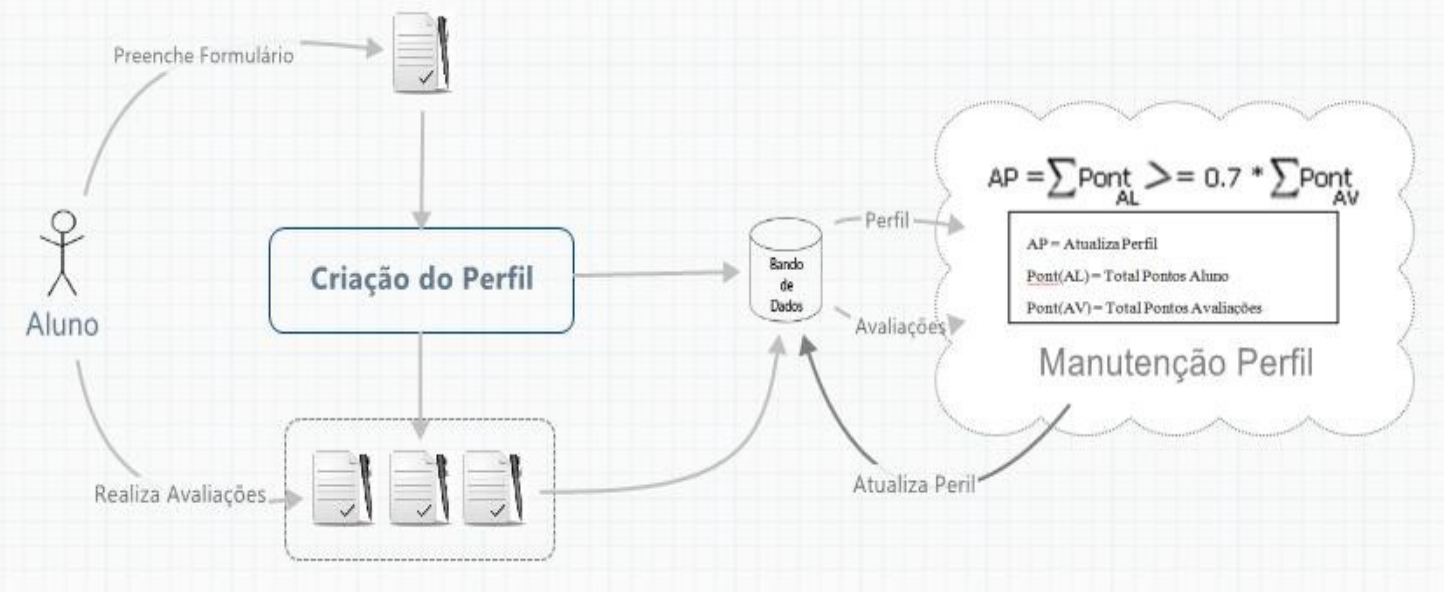

Figura 06: Criação e Manutenção do Perfil

\section{Objetos de Aprendizagem}

Atualmente, os Objetos de Aprendizagem (OA) podem ser considerados como recursos importantes no processo de ensino e aprendizagem, uma vez que nos fornecem a capacidade de simular e animar fenômenos, entre outras características, assim como, reutilizá-los em vários outros ambientes de aprendizagem. Eles podem ser localizados na Internet, através de repositórios, proporcionando, entre outras características, a redução de custos de produção de materiais educacionais (Santos et al 2007). Além disso, eles surgem como um recurso capaz de potencializar a reestruturação de práticas pedagógicas, criando novas maneiras de refletir sobre o uso da comunicação, da informação e da interação (Audino e Nascimento 2010).

Haughey e Muirhead (2005) citados por Cazella et al (2011), apontam que existem diversas definições para o termo OA e que algumas se concentram na parte "objeto" do termo, enquanto outros têm enfatizado o aspecto da "aprendizagem". Os 
autores ressaltam que os OAs não têm valor ou utilidade fora dos contextos de ensino, seu valor está na sua aplicação às configurações de sala de aula e ambientes online onde os professores podem ou não estar presentes.

Para Tarouco et al. (2004), Objetos de aprendizagem são materiais educacionais com objetivos pedagógicos que servem para apoiar o processo de ensino $\mathrm{e}$ aprendizagem, já Behar et al. (2009) citado por Audino e Nascimento (2010) entendem que os objetos de aprendizagem são qualquer material digital, como, por exemplo, textos, animações, vídeos, imagens, aplicações, páginas web, de forma isolada ou em combinação, com fins educacionais. Para a autora, trata-se de um instrumento autônomo, que pode ser utilizado como módulo de determinado conteúdo, bem como, de um conteúdo completo, podendo ainda ser incorporado a múltiplos aplicativos.

Para o contexto deste trabalho, Objetos de Aprendizagem apresentam-se como sendo questões específicas que comporão as avaliações individuais dos alunos, com base em suas competências em SQL.

\section{Mecanismo de Recomendação do WebSQL}

O WebSQL dispõe de duas abordagens diferentes para propor a recomendação aos alunos usuários do sistema, uma através da Colaboração, afim de proporcionar e incentivar a formação de grupos de estudos, com o objetivo do avanço coletivo da turma rumo a construção do conhecimento, e a outra baseada em Conteúdo, por meio da recomendação de questões específicas que comporão suas avaliações levando em consideração o perfil (competências) do aluno.

\subsection{Abordagem Colaborativa}

Nesse tipo de abordagem os usuários recebem recomendações com base nas preferências de outros usuários que possuem perfis semelhantes. Uma das tarefas do sistema é identificar os grupos de usuários com características semelhantes, chamados de grupos de pares ou grupos de vizinhos, processo esse chamado de geração da vizinhança (Sarwar et al., 2000). Esses grupos devem conter indivíduos com interesses ou comportamentos comuns (Cazella et al., 2010).

No WebSQL, a recomendação colaborativa apresenta-se através da indicação de outros alunos que tenham competências para ajudar o aluno alvo da recomendação, na solução de alguma questão que por ventura tenha dificuldades na resolução.

O sistema monitora a interação do aluno na resolução de suas atividades, controlando a quantidade de vezes que pode tentar para chegar à solução ótima de determinada questão, bem como a quantidade de vezes já tentadas; ao constatar que o aluno já utilizou 70\% das possibilidades e ainda não chegou à solução ideal, o sistema busca os alunos que já resolveram tal questão e que possuem o mesmo perfil do aluno alvo da indicação, levando em consideração a questão, a quantidade de vezes tentadas e se o aluno já chegou à solução ótima e indicam os cinco primeiros alunos encontrados através de uma lista contendo o nome e o e-mail dos alunos recomendados, tal recomendação pode ser vista na figura 07. 


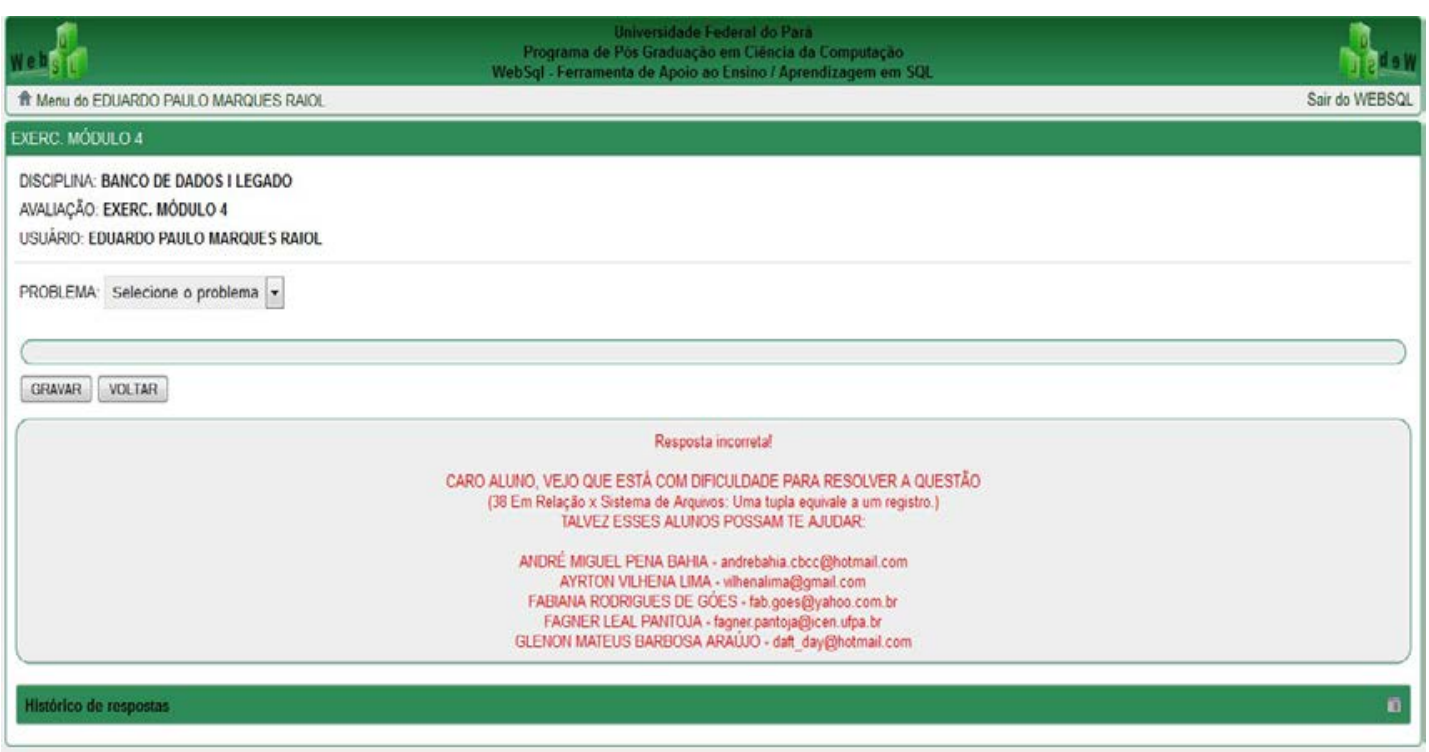

Figura 07: Recomendação Colaborativa para estímulo do trabalho em grupo

\subsection{Abordagem Baseada em Conteúdo}

Na técnica baseada em conteúdo os usuários recebem recomendações de itens com características similares às de outros itens adquiridos no passado. Para essa forma de recomendação é analisado o histórico da interação entre usuário e ambiente, com o objetivo de se obter quais produtos foram adquiridos pelos usuários no passado (Felfernig e Burke 2008).

No WebSQL essa técnica apresenta-se através da indicação de questões específicas, para compor a avaliação do aluno, baseadas na competência (perfil) do aluno em SQL; o professor inicialmente cadastra as questões no repositório de questões do ambiente por grupo (Objetivo, Discursivo ou SQL) e por nível de dificuldade (Baixa, Média ou Alta), ao gerar avaliações através da recomendação, o professor seleciona a quantidade de questões que deseja na avaliação e a turma, para o qual a avaliação será gerada, conforme verificamos na figura 08 .

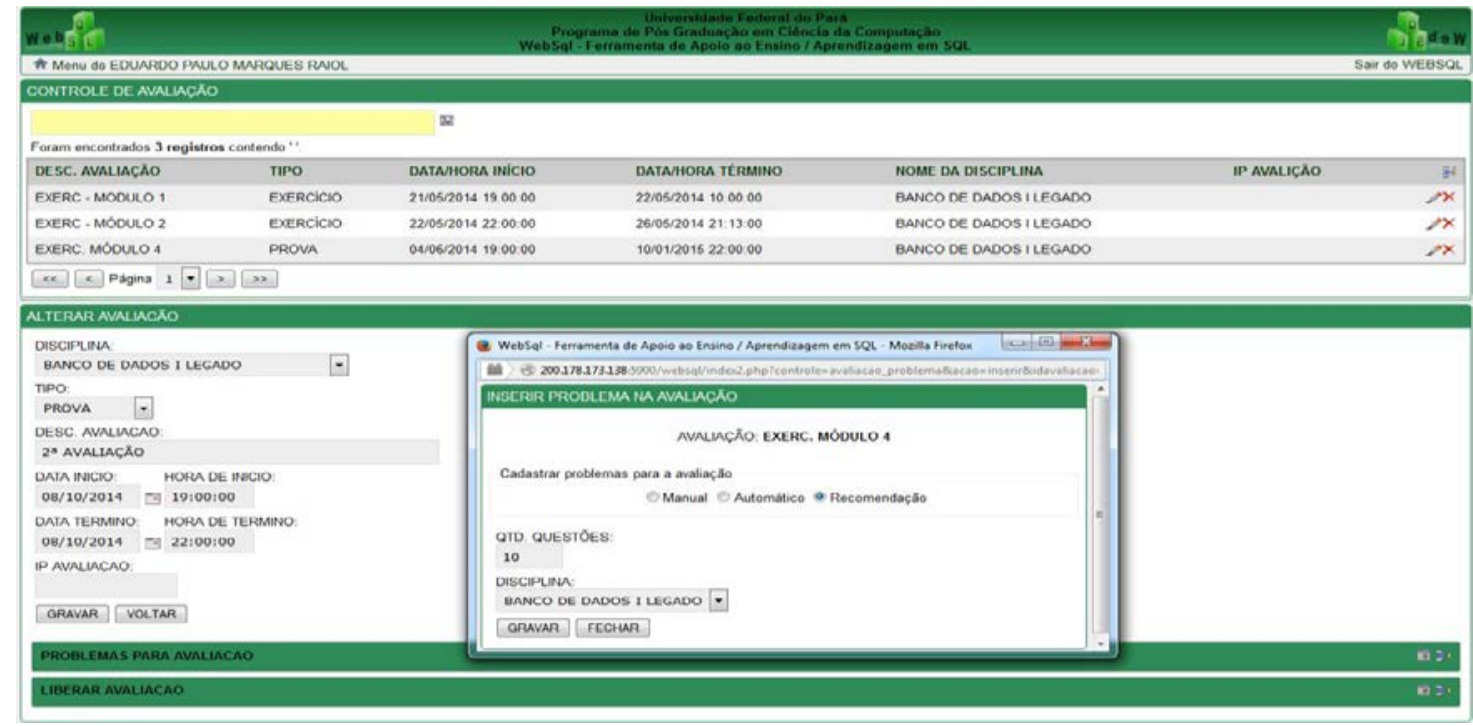

Figura 08: Geração de avaliação através da recomendação 
Ao gerar a avaliação, o módulo Recomendação obtém o perfil da cada aluno pertencente à turma selecionada, e filtra as questões que mais se enquadram ao perfil do aluno, montando avaliações personalizadas e individualizadas para cada aluno, conforme a arquitetura de recomendação da figura 09.

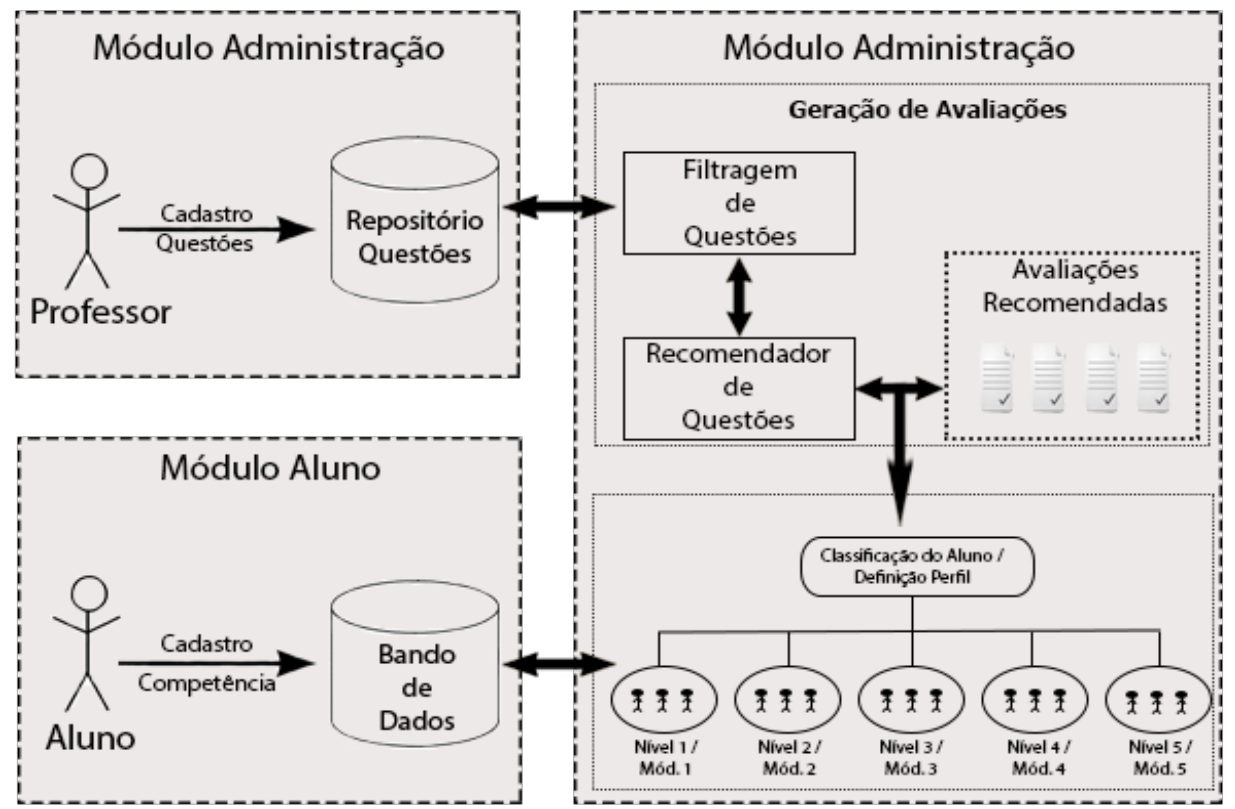

Figura 09: Arquitetura do módulo Recomendação de Questões

\section{Experimento}

Os testes e validação do WebSQL estão ocorrendo em dois momentos, no primeiro, já concluído, foram utilizados 10 alunos de graduação do $4^{\circ}$ semestre, do curso de Sistemas de Informação, na disciplina de Projeto de Banco de Dados, onde foi observado o comportamento do ambiente acerca da construção e manutenção dos perfis dos alunos e o processo de recomendação de questões, foram ainda realizadas correções de erros e implementações de novas solicitações dos usuários (Professores e Alunos). Foram criados, de forma explícita, através do preenchimento do formulário de competências, e mantidos de forma implícita, por meio do monitoramento das ações dos alunos, 10 perfis, tendo sido geradas 02 avaliações, através da recomendação, contendo 12 questões cada, onde obtiveram um total de 248 respostas às questões indicadas nas avaliações.

Quanto à avaliação do ambiente, verificou-se que o mesmo vem atendendo de forma satisfatória às necessidades pedagógicas dos professores e alunos, mas que estamos no início dos testes e ainda não é possível afirmar se atingimos ou não esse objetivo, observou-se também que a indicação das questões para comporem as avaliações dos alunos e a criação e manutenção do perfil, vem sendo feita de forma correta, bem como na indicação de alunos com potencial para colaborar com a construção do conhecimento do aluno alvo da recomendação, proporcionando e incentivando assim a formação de grupos de estudo.

No segundo momento, já iniciado, foi disponibilizado o acesso a mais duas turmas também de graduação, uma do curso de Análise e Desenvolvimento de Sistemas, 
na Disciplina de Banco de Dados, com 25 alunos, e a outra do curso de Ciência da Computação, na disciplina de Linguagem de Programação para Banco de Dados, com 50 Alunos, assim poderemos monitorar a criação e manutenção desses novos perfis, a fim de comprovar a eficácia do modelo de classificação do aluno utilizando o KNN.

Ao final teremos um quantidade expressiva de avaliações e perfis gerados, possibilitando assim uma análise mais criteriosa do ambiente, para então aplicarmos um questionário, no próprio ambiente, que serão investigados acerca da Usabilidade, Funcionalidade, Confiabilidade e Eficiência do WebSQL, fechando assim toda etapa de experimento da nossa proposta, para liberação efetiva da mesma para utilização nas instituições parceiras e outras que tenham interesse no uso do WebSQL.

\section{Considerações finais}

Este trabalho apresentou uma ferramenta para o apoio à aprendizagem da linguagem SQL, cujo principal objetivo foi descrever o processo de recomendação existente no WebSQL, destacando a capacidade de realizar recomendações aos alunos usuários do sistema de duas formas: a) uma baseada em conteúdo, através da geração de avaliações individualizadas com base no perfil (competência) do aluno; b) a outra de forma colaborativa, por meio da indicação de outros alunos com o mesmo perfil do aluno alvo da recomendação, cujo objetivo é o estímulo do trabalho em grupo.

Destaca-se ainda, a contribuição do trabalho para a prática pedagógica do professor, quanto ao ensino e aprendizagem da linguagem SQL, com o intuito de apoiar a criação de novas estratégias de ensino, a partir do uso do WebSQL para a construção do conhecimento dos alunos.

Como trabalhos futuros pretende-se expandir a recomendação, através da integração a repositórios de OA, também, para materiais didáticos, ou seja, além do aluno receber recomendação de questões que comporão suas avaliação e indicação de outros alunos com o mesmo perfil, receberá também recomendação de materiais que poderiam fortalecer e colaborar com a construção do seu conhecimento.

\section{Referências Bibliográficas}

Audino, F. D., Nascimento, R. S. Objetos de Aprendizagem - Diálogo entre conceitos e uma nova proposição aplicada à educação, in Revista Contemporânea de Educação, Vol. 5, n. 10, Jul/Dez 2010.

Barth, F., J. (2010) Modelando o perfil do usuário para a construção de sistemas de recomendação: um estudo teórico e estado da arte. Revista de Sistemas de Informação da FSMA, n. 6.

Cazella, S. C., Behar, P., Schneider, D., Silva, Ketia Kellen, Freitas, R., Recomendando objetos de aprendizagem baseado em competências em ead. Revista Renote Novas Tecnologias na Educação, 2011, Disponível em: <http://seer.ufrgs.br/renote/article/view/25123> Acessado em: 14 de julho de 2014.

Cazella, S. C.; Nunes, M. A. S. N.; Reategui, E. B., A Ciência da Opinião: Estado da arte em Sistemas de Recomendação. In XXX Congresso da SBC, Belo Horizonte, 2010. 
Felferning, A.; Burke, R. D., Constraint-based recommender systems: technologies and research issues. ACM International Conference Proceeding Series. Vol. 342, 2008.

Fleury, A., Fleury, M., T. Construindo o conceito de competência. RAC, Edição Especial 2001. Disponível em: <http://www.scielo.br/pdf/rac/v5nspe/ v5nspea10.pdf $>$. Acessado em: 25 agosto 2014.

Lazzaroto, L. L., Braga, J. L., Oliveira, A. P., Passos, F. J. V., A educação em ambientes virtuais: proposição de recursos computacionais para aumentar a eficiência do processo ensino-aprendizado, in Revista Brasileira de Informática na Educação, Volume 19, Número 2, 2011.

Pereira Junior, J.C.R., Rapkiewicz, C., O Processo de Ensino-Aprendizagem de Fundamentos de Programação: Uma Visão Crítica da Pesquisa no Brasil, WEI RJES, 2004.

Perrenoud, P. (1999) Construir as competências desde a escola. Artmed, Porto Alegre.

Prior, J. C. and R. Lister. The backwash effect on sql skills grading. In Proceedings of the 9th Annual SIGCSE Conference on innovation and Technology in Computer Science Education, 2004.

Santos, L., M., A., Flôres, M., L., P., Tarouco, L., M., R. Objetos de Aprendizagem: teoria instrutiva apoiada por computador. RENOTE - Revista Novas Tecnologias na Educação. Porto Alegre, v. 6, v. 2, 2007

Sarwar, B.; Karypis, G.; Konstan, N., J., Riedl, J., Analysis of Recommendation Algorithms for E-Commerce. Electronic Commerce, 2000, Disponível em <http://dl.acm.org/citation.cfm?id=352887>. Acesso: 10 jun. 2014.

Tarouco, L. M. R., Fabre, M. C. J. M., Konrath, M. L. P., Grando, A. R., Objetos de Aprendizagem para M-Learning. In: Congresso Nacional de Tecnologia da Informação e Comunicação (SUCESU). Florianópolis. Anais do Congresso Nacional de Tecnologia da Informação e Comunicação. Florianópolis, 2004. Disponível em: $<$ http://www.cinted.ufrgs.br/CESTA/objetosdeaprendizagem_sucesu.pdf $>$. Acesso em: 07 jun. 2014. 